\title{
MIXED BOUNDARY VALUE PROBLEM FOR AN ANISOTROPIC THERMOELASTIC HALF-SPACE CONTAINING THIN INHOMOGENEITIES
}

\author{
Heorhiy SULYM*, laroslav PASTERNAK**, Mariia SMAL**, Andrii VASYLYSHYN"** \\ *Bialystok University of Technology, ul. Wiejska 45C, 15-351 Bialystok, Poland \\ ${ }^{*}$ *Lutsk National Technical University, Lvivska Str. 75, 43018 Lutsk, Ukraine \\ ${ }^{* *}$ Ivan Franko National University of Lviv, Universytetska Str. 1, 79000 Lviv, Ukraine
}

h.sulym@pb.edu.pl, yaroslav.pasternak@gmail.com, m.smal@Intu.edu.ua, vasylyshyn.c.h@gmail.com

received 16 May 2019, revised 6 December 2019, accepted 13 December 2019

\begin{abstract}
The paper presents a rigorous and straightforward approach for obtaining the 2D boundary integral equations for a thermoelastic half-space containing holes, cracks and thin foreign inclusions. It starts from the Cauchy integral formula and the extended Stroh formalism which allows writing the general solution of thermoelastic problems in terms of certain analytic functions. In addition, with the help of it, it is possible to convert the volume integrals included in the equation into contour integrals, which, in turn, will allow the use of the method of boundary elements. For modelling of solids with thin inhomogeneities, a coupling principle for continua of different dimensions is used. Applying the theory of complex variable functions, in particular, Cauchy integral formula and Sokhotski-Plemelj formula, the Somigliana type boundary integral equations are constructed for thermoelastic anisotropic half-space. The obtained integral equations are introduced into the modified boundary element method. A numerical analysis of the influence of boundary conditions on the half-space boundary and relative rigidity of the thin inhomogeneity on the intensity of stresses at the inclusions is carried out.
\end{abstract}

Keywords: thermoelasticity, anisotropic half-space, boundary element method, thin inclusion, crack, stress intensity factors, Stroh formalism

\section{INTRODUCTION}

Modern composite materials are widely used in engineering structures due to their useful properties (Woo, 2011). To a large extent, most of them are anisotropic. It is also clear that the internal structure of most of them is not perfect. Since material fracture generally initiates at various defects, there is a need for the development of analytical and numerical approaches for the analysis of internal physical and mechanical fields and the strength and reliability of the structural elements made of composite materials.

In most cases, modelling of bulky structural elements can be reduced to the analysis of semi-infinite solids, for example, halfspace, since it is essential to estimate the influence of interaction of internal inhomogeneities with the boundary of a solid. A threedimensional model for solving steady-state heat conduction in a semi-infinite domain containing an elementary cuboidal inhomogeneity was established by Yang et al. (2019). Three-dimensional exact fundamental solutions of the thermoelastic field in a transversely isotropic elastic medium weakened by a half infinite plane crack subjected to a pair of point thermal loadings symmetrically acting on the crack surface were presented by Li (2012). Sherief et al. (2014) applied the fractional order theory of thermoelasticity to a two-dimensional problem for a half-space. Şeremet (2011) derived the exact Green's function and a Poisson-type integral formula for a boundary-value problem (BVP) for a thermoelastic wedge, half-space and quarter-space. For solving twodimensional thermoelastic crack problems, Chen et al. (2016) used singular edge-based smoothed finite element method (ESFEM).

The jump function method is convenient and effective in simulation of thin inhomogeneities (Sulym, 2007). The idea of this method is that the inclusion as a geometric object is excluded from consideration, and its effect is reduced to introduction of certain functions of the jump of physical and mechanical fields in the medium while passing the median surface of the thin-walled inhomogeneity.

The boundary element method (BEM) perfectly suits for solving the stress concentration and fracture mechanics problems due to its high precision and efficiency (Bozhydarnyk et al., 2011; Hou, 2011; Qin, 1999; Shiah, 2000). Tokovyy and Ma (2009) obtained the Volterra integral equations of thermoelasticity for the orthotropic plane, half-plane and a strip. However, in the presence of thermal effects, there are additional volume integral terms in integral equations that fully reduce the advantages of BEM. In the case of isotropic solids, these volume integrals can be easily converted to boundary ones (Mukherjee, 1999). However, in the case of anisotropy, transformation of the volume integral into the contour one is a difficult task. For the first time, in the case of a plane thermoelasticity problem, the temperature volume integral was reduced to the contour one in the real domain in Pasternak (2012).

The abovementioned works consider mainly half-space with traction-free thermally insulated surface. However, in most of the engineering problems, especially those of contact mechanics, mixed thermomechanical boundary conditions should be considered, that is, at the half-space boundary given are some components of traction vector and some components of displacement vector. For example, if the half-space boundary $\mathrm{x}_{2}=0$ rests on the smooth rigid basement, the component $\mathrm{u}_{2}$ of displacement vector and the component $t_{1}$ of traction vector are zero. Such problems for thermoelastic anisotropic half-space are not found in scientific literature. 
In this paper, the methods based on the complex variable calculus, the Stroh formalism, the jump function method and the BEM (Pasternak, 2012; Pasternak et al., 2013) are used to obtain new integral formulas and equations for anisotropic thermoelastic half-space with holes, cracks and thin deformable inclusions, taking into account all possible mixed mechanical and thermal boundary conditions on its boundary.

\section{GOVERNING EQUATIONS OF THERMOELASTICITY}

Consider a solid resting in a fixed rectangular coordinate system $O x_{1} x_{2} x_{3}$. According to Hwu (2010), the equilibrium equation, the equation of thermal balance, as well as the constitutive equations of plain strain of a linearly thermoelastic body and of plane stationary heat conductivity are written as:

$\sigma_{i j, j}=0, h_{i, i}=0,(i, j=1,2,3)$;

$\sigma_{i j}=C_{i j k m} \varepsilon_{k m}-\beta_{i j} \theta, h_{i}=-k_{i j} \theta_{, j}$,

where $\varepsilon_{i j}=\left(u_{i, j}+u_{j, i}\right) / 2$ is a strain tensor, $\sigma_{i j}$ is a stress tensor, $h_{i}$ is a heat flux vector, $u_{i}$ is a displacement vector, $\theta$ is the change in temperature compared with the reference one, $C_{i j k m}$ are the elastic moduli, $k_{i j}$ are the heat conduction coefficients, $\beta_{i j}=C_{i j k m} \alpha_{k m}(i, j, k, m=1, \ldots, 3)$ are the thermal moduli and $\alpha_{i j}$ are the thermal expansion coefficients. The tensors $C_{i j k m}, k_{i j}, \alpha_{i j}$ and $\beta_{i j}$ are fully symmetric. Here and further, the Einstein summation convention is used. Using the generalised Stroh formalism, it is possible to derive the following dependences:

$\theta=2 \operatorname{Re}\left\{g^{\prime}\left(z_{t}\right)\right\}, \vartheta=2 k_{t} \operatorname{Im}\left\{g^{\prime}\left(z_{t}\right)\right\}, h_{1}=-\vartheta_{, 2}, h_{2}=\vartheta_{, 1}, k_{t}=$ $\sqrt{k_{11} k_{22}-k_{12}{ }^{2}}, \mathbf{u}=2 \operatorname{Re}\left[\mathbf{A f}\left(z_{*}\right)+\mathbf{c} g\left(z_{t}\right)\right], \boldsymbol{\varphi}=2 \operatorname{Re}\left[\mathbf{B f}\left(z_{*}\right)+\right.$ $\left.\mathbf{d} g\left(z_{t}\right)\right], \quad \sigma_{i 1}=-\varphi_{i, 2}, \quad \sigma_{i 2}=\varphi_{i, 1} ; \quad z_{t}=x_{1}+p_{t} x_{2}, \quad z_{\alpha}=x_{1}+$ $p_{\alpha} x_{2}, \mathbf{f}\left(z_{*}\right)=\left[F_{1}\left(z_{1}\right), F_{2}\left(z_{2}\right), F_{3}\left(z_{3}\right)\right]^{\mathrm{T}}$,

where $\vartheta$ is a heat flux function, $F_{\alpha}\left(z_{\alpha}\right)$ and $g\left(z_{t}\right)$ are the complex analytic functions with respect to their arguments and the complex constant $p_{t}$ is a root (with a positive imaginary part) of the characteristic equation for heat conduction

$k_{22} p_{t}^{2}+2 k_{12} p_{t}+k_{11}=0$.

Constant complex matrices $\mathbf{A}, \mathbf{B}$, vectors $\mathbf{c}$, $\mathbf{d}$ and scalars $p_{\alpha}(\alpha=1,2,3)$ are determined from the extended Stroh eigenvalue problem.

The relationship between the Stroh's complex potentials and vector functions of displacements and stresses is given by the following relations:

$\mathbf{f}\left(z_{*}\right)=\mathbf{B}^{\mathrm{T}} \mathbf{u}+\mathbf{A}^{\mathrm{T}} \boldsymbol{\varphi}-\boldsymbol{B}^{\mathrm{T}} \mathbf{u}^{t}-\mathbf{A}^{\mathrm{T}} \boldsymbol{\varphi}^{t}$,

$\mathbf{u}^{\mathrm{t}}=2 \operatorname{Re}\left\{\mathbf{c} g\left(z_{t}\right)\right\}, \varphi^{\mathrm{t}}=2 \operatorname{Re}\left\{\mathbf{d} g\left(z_{t}\right)\right\}$.

Based on Eq. (3), one can derive the following relation between the function $g^{\prime}\left(z_{t}\right)$, temperature and heat flux function

$g^{\prime}\left(z_{t}\right)=\frac{1}{2}\left(\theta+i \frac{\vartheta}{k_{t}}\right)$.

\section{DERIVATION OF INTEGRAL FORMULAE FOR THE STROH COMPLEX FUNCTIONS}

For thermoelastic half-space $x_{2}>0$ containing a system of holes bounded with smooth closed contours $\Gamma=\cup_{i} \Gamma_{i}$, the following dependencies are derived: $\mathbf{f}\left(z_{*}\right)=\frac{1}{2 \pi i} \int_{\Gamma}\left(\frac{d \tau_{*}}{\tau_{*}-z_{*}}\right) \mathbf{f}\left(\tau_{*}\right)+\frac{1}{2 \pi i} \int_{-\infty}^{\infty}\left\langle\frac{d x_{1}}{x_{1}-z_{*}}\right\rangle \mathbf{f}\left(x_{1}\right),\left(\operatorname{Im} z_{*}>0\right),(6)$ where $\left\langle F\left(z_{*}\right)\right\rangle=\operatorname{diag}\left[F_{1}\left(z_{1}\right), F_{2}\left(z_{2}\right), F_{3}\left(z_{3}\right)\right]$.

It is obvious that outside the half-space, Cauchy integrals (6) are equal to zero. Therefore, for external points $\bar{Z}_{*}\left(\operatorname{Im} Z_{*}>0\right)$, Eq. (6) is writes as:

$\frac{1}{2 \pi i} \int_{\Gamma}\left(\frac{d \tau_{*}}{\tau_{*}-\bar{z}_{*}}\right) \mathbf{f}\left(\tau_{*}\right)+\frac{1}{2 \pi i} \int_{-\infty}^{\infty}\left\langle\frac{d x_{1}}{x_{1}-\bar{z}_{*}}\right\rangle \mathbf{f}\left(x_{1}\right)=0,\left(\operatorname{Im} z_{*}>0\right)$.

Integral representations in Eqs (6) and (7) include linear integrals along the infinite half-space boundary that are inconvenient for further calculations. To exclude them from consideration, we will use four different types of boundary conditions on the half-space boundary:

$\vartheta\left(x_{1}\right)=0\left(x_{2}=0\right)$;

$\theta\left(x_{1}\right)=0\left(x_{2}=0\right)$;

$u_{1}\left(x_{1}\right)=0, \varphi_{2}\left(x_{1}\right)=0\left(x_{2}=0\right)$;

$u_{2}\left(x_{1}\right)=0, \varphi_{1}\left(x_{1}\right)=0\left(x_{2}=0\right)$.

\subsection{Heat conduction}

According to the boundary conditions in Eq. (8a) and Eq. (5), the integral formula for the function $g^{\prime}\left(z_{t}\right)$ can be rewritten as follows:

$g^{\prime}\left(z_{t}\right)=\frac{1}{2 \pi i} \int_{\Gamma} \frac{g r\left(\tau_{t}\right) d \tau_{t}}{\tau_{t}-z_{t}}+\frac{1}{4 \pi k_{t}} \int_{-\infty}^{\infty} \frac{\vartheta\left(x_{1}\right) d x_{1}}{x_{1}-z_{t}}$

Having calculated the complex conjugate expression for the Cauchy integral and using Eqs (6) and (5), we obtain the integral value along the half-space boundary:

$\frac{1}{4 \pi i} \int_{-\infty}^{\infty} \frac{\theta\left(x_{1}\right) d x_{1}}{x_{1}-z_{t}}=-\frac{1}{2 \pi i} \int_{\Gamma} \frac{\overline{g^{\prime}\left(\tau_{t}\right)} d \bar{\tau}_{t}}{\bar{\tau}_{t}-z_{t}}$.

Substituting Eq. (10) into (9), one obtains the integral formula for a complex function $g^{\prime}\left(z_{t}\right)$.

$g^{\prime}\left(z_{t}\right)=\frac{1}{2 \pi i}\left[\int_{\Gamma} \frac{g \prime\left(\tau_{t}\right) d \tau_{t}}{\tau_{t}-z_{t}}-\int_{\Gamma} \frac{\overline{g r\left(\tau_{t}\right)} d \bar{\tau}_{t}}{\bar{\tau}_{t}-z_{t}}\right]$.

Using Eq. (5), we can construct an integral representation of a function $g^{\prime}\left(z_{t}\right)$ using the boundary values of physical parameters $\vartheta$ and $\theta$ :

$g^{\prime}\left(z_{t}\right)=\frac{1}{4 \pi i}\left[\int_{\Gamma} \frac{\left(\theta+i \frac{\vartheta}{k_{t}}\right) d \tau_{t}}{\tau_{t}-z_{t}}-\int_{\Gamma} \frac{\left(\theta-i \frac{\vartheta}{k_{t}}\right) d \bar{\tau}_{t}}{\bar{\tau}_{t}-z_{t}}\right]$.

Eq. (12) can be reduced to the first-order curvilinear integrals:

$g^{\prime}\left(z_{t}\right)=-\frac{1}{4 \pi i} \int_{\Gamma}\left[\frac{n_{2}(s)-p_{t} n_{1}(s)}{\tau_{t}(s)-z_{t}}-\frac{n_{2}(s)-\overline{p_{t}} n_{1}(s)}{\bar{\tau}_{t}(s)-z_{t}}\right] \theta(s) d s$

$+\frac{1}{4 \pi k_{t}} \int_{\Gamma}\left[\ln \left(\tau_{t}(s)-z_{t}\right)+\ln \left(\bar{\tau}_{t}(s)-z_{t}\right)\right] h_{n}(s) d s$.

Based on Eqs (8) and (13), we obtain integral representations for temperature and heat flux at an arbitrary point $\xi$ in the halfspace $x_{2}>0$

$\theta(\xi)=2 \operatorname{Re}\left\{g^{\prime}\left(Z_{t}(\xi)\right)\right\}$

$=\int_{\Gamma}\left[\Theta^{h s *}(\mathbf{x}, \xi) h_{n}(\mathbf{x})-H^{h s *}(\mathbf{x}, \xi) \theta(\mathbf{x})\right] d s(\mathbf{x})$,

$h_{i}(\xi)=2 k_{t} \operatorname{Im}\left\{\left(\delta_{2 i}-\delta_{1 i} p_{t}\right) g^{\prime \prime}\left(Z_{t}(\xi)\right)\right\}$

$=\int_{\Gamma} \Theta_{i}^{h s * *}(\mathbf{x}, \xi) h_{n}(\mathbf{x}) d \Gamma(\mathbf{x})-\int_{\Gamma} H_{i}^{h s * *}(\mathbf{x}, \xi) \theta(\mathbf{x}) d s(\mathbf{x})$, 
$\Theta^{h s *}(\mathbf{x}, \xi)=\frac{1}{2 \pi k_{t}}\left[\ln \left|Z_{t}(\mathbf{x}-\xi)\right|+\ln \left|\bar{Z}_{t}(\mathbf{x})-Z_{t}(\xi)\right|\right]$,

$\mathrm{H}^{h s *}(\mathbf{x}, \xi)=\frac{1}{2 \pi} \operatorname{Im}\left\{\frac{n_{2}(\mathbf{x})-p_{t} n_{1}(\mathbf{x})}{Z_{t}(\mathbf{x}-\xi)}-\frac{n_{2}(\mathbf{x})-\bar{p}_{t} n_{1}(\mathbf{x})}{\bar{Z}_{t}(\mathbf{x})-\overline{Z_{t}(\xi)}}\right\}$

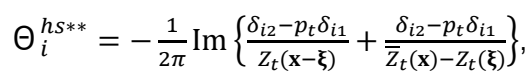

$\mathrm{H}_{i}^{h s * *}=-\frac{k_{t}}{2 \pi} \operatorname{Re}\left\{\left(\delta_{i 2}-p_{t} \delta_{i 1}\right)\right.$

$\left.\times\left[\frac{n_{2}(\mathbf{x})-p_{t} n_{1}(\mathbf{x})}{\left[Z_{t}(\mathbf{x}-\xi)\right]^{2}}-\frac{n_{2}(\mathbf{x})-\bar{p}_{t} n_{1}(\mathbf{x})}{\left[\bar{Z}_{t}(\mathbf{x})-Z_{t}(\xi)\right]^{2}}\right]\right\}$.

Here, $Z_{*}(\mathbf{x})=x_{1}+p_{*} x_{2}$.

\subsection{Thermoelasticity}

Using the boundary conditions (8c) and (8d) and representation of complex potentials in Eq. (4), we can rewrite the integral along the boundary of the half-space in Eq. (6) as follows:

$\int_{-\infty}^{\infty}\left(\frac{d x_{1}}{x_{1}-z_{*}}\right) \mathbf{f}\left(x_{1}\right)=\int_{-\infty}^{\infty}\left(\frac{d x_{1}}{x_{1}-z_{*}}\right)\left[\mathbf{B}^{\mathrm{T}} \mathbf{u}+\mathbf{A}^{\mathrm{T}} \boldsymbol{\varphi}\right.$

$-\mathbf{B}^{\mathrm{T}} \mathbf{u}^{t}\left(x_{1}\right)-\mathbf{A}^{\mathrm{T}} \boldsymbol{\varphi}^{t}\left(x_{1}\right)$.

As a result of integration by parts of Eq. (20) and the limitation of functions $\mathbf{u}^{t}\left(x_{1}\right)$ and $\boldsymbol{\varphi}^{t}\left(x_{1}\right)$, we obtain the following equation.

$\int_{-\infty}^{\infty}\left\langle\frac{d x_{1}}{x_{1}-z_{*}}\right\rangle \mathbf{f}\left(x_{1}\right)=\int_{-\infty}^{\infty}\left\langle\frac{d x_{1}}{x_{1}-z_{*}}\right\rangle \mathbf{A}^{\mathrm{T}} \boldsymbol{\varphi}+\int_{-\infty}^{\infty}\left\langle\frac{d x_{1}}{x_{1}-z_{*}}\right\rangle \mathbf{B}^{\mathrm{T}} \mathbf{u}$

$+\int_{-\infty}^{\infty}\left\langle\ln \left(x_{1}-z_{*}\right)\right\rangle\left[\mathbf{B}^{\mathrm{T}} \frac{\partial \mathbf{u}^{\mathrm{t}}\left(x_{1}\right)}{\partial x_{1}}+\mathbf{A}^{\mathrm{T}} \frac{\partial \boldsymbol{\varphi}^{\mathrm{t}}\left(x_{1}\right)}{\partial x_{1}}\right] d x_{1}$.

Now consider that, according to Eqs (5) and (6) and the boundary conditions in Eqs (8a) and (8c):

$\mathbf{B}^{\mathrm{T}} \frac{\partial \mathbf{u}^{t}\left(x_{1}\right)}{\partial x_{1}}+\mathbf{A}^{\mathrm{T}} \frac{\partial \boldsymbol{\varphi}^{t}\left(x_{1}\right)}{\partial x_{1}}=\mathbf{m} \theta$,

where

$\mathbf{m}=\mathbf{B}^{\mathrm{T}} \operatorname{Re}[\mathbf{c}]+\mathbf{A}^{\mathrm{T}} \operatorname{Re}[\mathbf{d}]$.

Therefore, the Cauchy integral in Eq. (21) is written as

$\int_{-\infty}^{\infty}\left\langle\frac{d x_{1}}{x_{1}-z_{*}}\right\rangle \mathbf{f}\left(x_{1}\right)=\int_{-\infty}^{\infty}\left\langle\frac{d x_{1}}{x_{1}-z_{*}}\right\rangle \mathbf{A}^{\mathrm{T}} \boldsymbol{\varphi}+\int_{-\infty}^{\infty}\left\langle\frac{d x_{1}}{x_{1}-z_{*}}\right\rangle \mathbf{B}^{\mathrm{T}} \mathbf{u}$

$+\int_{-\infty}^{\infty}\left\langle\ln \left(x_{1}-z_{*}\right)\right\rangle \mathbf{m} \theta\left(x_{1}\right) d x_{1}$.

Substituting Eq. (24) in Eq. (7), we can write:

$\int_{-\infty}^{\infty}\left\langle\frac{d x_{1}}{x_{1}-\bar{z}_{*}}\right\rangle\left[\mathbf{A}^{\mathrm{T}} \boldsymbol{\varphi}+\mathrm{B}^{\mathrm{T}} \mathbf{u}\right]=-\int_{\Gamma}\left(\frac{d \tau_{*}}{\tau_{*}-\bar{z}_{*}}\right\rangle \mathbf{f}\left(\tau_{*}\right)$

$-\int_{-\infty}^{\infty}\left\langle\ln \left(x_{1}-\bar{z}_{*}\right)\right\rangle \mathbf{m} \theta\left(x_{1}\right) d x_{1}$.

Let us introduce the notation $\mathbf{A}^{\mathrm{T}} \boldsymbol{\varphi}+\mathbf{B}^{\mathrm{T}} \mathbf{u}=\mathbf{C X}$, where $\mathbf{X}=\left(\begin{array}{l}\mathbf{u} \\ \boldsymbol{\varphi}\end{array}\right), \mathbf{C}$ is a square matrix that will be formed on the basis of the matrix $\left(\mathbf{B}^{\mathrm{T}} \mathbf{A}^{\mathrm{T}}\right)$.

Now we can rewrite Eq. (25) in a slightly different manner:

$$
\begin{aligned}
\int_{-\infty}^{\infty}\left\langle\frac{d x_{1}}{x_{1}-\bar{z}_{*}}\right\rangle\left[\mathbf{A}^{\mathrm{T}} \boldsymbol{\varphi}+\mathbf{B}^{\mathrm{T}} \mathbf{u}\right]=\int_{-\infty}^{\infty}\left\langle\frac{d x_{1}}{x_{1}-\bar{z}_{*}}\right\rangle \mathbf{C X} \\
=-\int_{\Gamma}\left\langle\frac{d \tau_{*}}{\tau_{*}-\bar{z}_{*}}\right\rangle \mathbf{f}\left(\tau_{*}\right)-\int_{-\infty}^{\infty}\left\langle\ln \left(x_{1}-\bar{z}_{*}\right)\right\rangle \mathbf{m} \theta\left(x_{1}\right) d x_{1}
\end{aligned}
$$

or in complex conjugate form

$\int_{-\infty}^{\infty}\left(\frac{d x_{1}}{x_{1}-z_{*}}\right) \overline{\mathbf{C}} \mathbf{X}=\int_{-\infty}^{\infty}\left(\frac{d x_{1}}{x_{1}-\bar{z}_{*}}\right)\left[\overline{\mathbf{A}}^{\mathrm{T}} \boldsymbol{\varphi}+\overline{\mathbf{B}}^{\mathrm{T}} \mathbf{u}\right]$
$=-\int_{\Gamma}\left\langle\frac{d \bar{\tau}_{*}}{\overline{\bar{\tau}_{*}-z_{*}}}\right\rangle \overline{\mathbf{f}\left(\tau_{*}\right)}-\int_{-\infty}^{\infty}\left\langle\ln \left(x_{1}-z_{*}\right)\right\rangle \overline{\mathbf{m}} \theta\left(x_{1}\right) d x_{1}$.

Accounting for the Stroh orthogonality relations, the first integral on the right side of Eq. (24) can be represented as

$\int_{-\infty}^{\infty}\left\langle\frac{d x_{1}}{x_{1}-z_{*}}\right\rangle\left[\mathbf{A}^{\mathrm{T}} \boldsymbol{\phi}+\mathbf{B}^{\mathrm{T}} \mathbf{u}\right]=\int_{-\infty}^{\infty}\left\langle\frac{d x_{1}}{x_{1}-z_{*}}\right\rangle \mathbf{C X}$

$=-\sum_{\beta=1}^{3} \mathbf{I}_{\beta} \mathbf{C} \overline{\mathbf{C}}^{-1} \int_{-\infty}^{\infty}\left(\frac{d x_{1}}{x_{1}-z_{\beta}}\right) \overline{\mathbf{C}} \mathbf{X}$.

Here, $\mathbf{I}_{1}=\operatorname{diag}[1,0,0], \mathbf{I}_{2}=\operatorname{diag}[0,1,0]$ and $\mathbf{I}_{3}=\operatorname{diag}[0,0,1]$.

Substituting Eq. (26) into Eq. (27) and taking into account the value received, Eq. (24) takes the form

$\int_{-\infty}^{\infty}\left(\frac{d x_{1}}{x_{1}-z_{*}}\right) \mathbf{f}\left(x_{1}\right)=\sum_{\beta=1}^{3} \int_{\Gamma}\left(\frac{d \bar{\tau}_{\beta}}{\bar{\tau}_{\beta}-z_{*}}\right) \mathbf{C}^{-1} \overline{\mathbf{C}} \mathbf{I}_{\beta} \overline{\mathbf{f}\left(\tau_{*}\right)}$

$+\int_{-\infty}^{\infty}\left\langle\ln \left(x_{1}-z_{*}\right)\right\rangle\left[\mathbf{m}+\mathbf{C}^{-1} \overline{\mathbf{C}} \overline{\mathbf{m}}\right] \theta\left(x_{1}\right) d x_{1}$,

and integrating Eq. (10) gives

$\int_{-\infty}^{\infty}\left\langle\ln \left(x_{1}-z_{*}\right)\right\rangle \theta\left(x_{1}\right) d x_{1}=-2 \int_{\Gamma}\left\langle\ln \left(\bar{\tau}_{t}-z_{*}\right)\right\rangle \overline{g^{\prime}\left(\tau_{t}\right)} d \bar{\tau}_{t}$.

Therefore, the integral formula in Eq. (7) can be written in the form which does not contains integrals along the boundary of the half-space

$\mathbf{f}\left(z_{*}\right)=\frac{1}{2 \pi i}\left[\int_{\Gamma}\left(\frac{d \tau_{*}}{\tau_{*}-z_{*}}\right) \mathbf{f}\left(\tau_{*}\right)+\sum_{\beta=1}^{3} \int_{\Gamma}\left\langle\frac{d \bar{\tau}_{\beta}}{\bar{\tau}_{\beta}-z_{*}}\right\rangle \mathbf{C}^{-1} \overline{\mathbf{C}} \mathbf{I}_{\beta} \overline{\mathbf{f}\left(\tau_{*}\right)}\right.$

$\left.-2 \int_{\Gamma}\left\langle\ln \left(\bar{\tau}_{t}-z_{*}\right)\right\rangle\left[\mathbf{m}+\mathbf{C}^{-1} \overline{\mathbf{C}} \overline{\mathbf{m}}\right] d \bar{\tau}_{t}\right]$.

Using Eqs (4) and (5), the integral formula in Eq. (30) takes the form:

$\mathbf{f}\left(z_{*}\right)=\frac{1}{2 \pi i}\left[-\int_{\Gamma}\left[\mid \frac{n_{2}(s)-p_{*} n_{1}(s)}{\tau_{*}(s)-z_{*}}\right) \mathbf{B}^{\mathrm{T}}\right.$

$\left.+\sum_{\beta=1}^{3}\left(\frac{n_{2}(s)-\bar{p}_{\beta} n_{1}(s)}{\bar{\tau}_{\beta}(s)-z_{*}}\right) \mathbf{C}^{-1} \overline{\mathbf{C}}_{\beta} \overline{\mathbf{B}}^{\mathrm{T}}\right] \mathbf{u}(s) d s$

$+\int_{\Gamma}\left[\left\langle\ln \left(\tau_{*}(s)-z_{*}\right)\right\rangle \mathbf{A}^{\mathrm{T}}\right.$

$\left.+\sum_{\beta=1}^{3}\left\langle\ln \left(\bar{\tau}_{\beta}(s)-z_{*}\right)\right\rangle \mathbf{C}^{-1} \overline{\mathbf{C}} \mathbf{I}_{\beta} \overline{\mathbf{A}}^{\mathrm{T}}\right] \mathbf{t}(s) d s$

$-\int_{\Gamma}\left\langle\ln \left(\tau_{*}(s)-z_{*}\right)\right\rangle\left(\mathbf{A}^{\mathrm{T}} \operatorname{Re}\left[\mathbf{d}\left(n_{2}-n_{1} p_{t}\right)\right]\right.$

$\left.+\mathbf{B}^{\mathrm{T}} \operatorname{Re}\left[\mathbf{c}\left(n_{2}-n_{1} p_{t}\right)\right]\right) \theta(s) d s$

$-\int_{\Gamma} \sum_{\beta=1}^{3}\left\langle\ln \left(\bar{\tau}_{\beta}(s)-z_{*}\right)\right\rangle \mathbf{C}^{-1} \overline{\mathbf{C}} \mathbf{I}_{\beta}\left(\overline{\mathbf{A}}^{\mathrm{T}} \operatorname{Re}\left[\mathbf{d}\left(n_{2}-n_{1} p_{t}\right)\right]\right.$

$\left.+\overline{\mathbf{B}}^{\mathrm{T}} \operatorname{Re}\left[\mathbf{c}\left(n_{2}-n_{1} p_{t}\right)\right]\right) \theta(s) d s$

$-\int_{\Gamma}\left\langle\ln \left(\bar{\tau}_{t}(s)-z_{*}\right)\right\rangle\left[n_{2}(s)-\bar{p}_{t} n_{1}(s)\right]\left[\mathbf{m}+\mathbf{C}^{-1} \overline{\mathbf{C}} \overline{\mathbf{m}}\right] \theta(s) d s$

$-\frac{1}{k_{t}} \int_{\Gamma}\left\langle f^{*}\left(\tau_{*}(s)-z_{*}\right)\right\rangle\left(\mathbf{A}^{\mathrm{T}} \operatorname{Im}[\mathbf{d}]+\mathbf{B}^{\mathrm{T}} \operatorname{Im}[\mathbf{c}]\right) h_{n}(s) d s$

$+\frac{1}{k_{t}} \int_{\Gamma}\left\langle f^{*}\left(\bar{\tau}_{t}-z_{*}\right)\right\rangle\left[\mathbf{m}+\mathbf{C}^{-1} \overline{\mathbf{C}} \overline{\mathbf{m}}\right] h_{n}(s) d s$

$-\frac{1}{k_{t}} \int_{\Gamma} \sum_{\beta=1}^{3}\left\langle f^{*}\left(\bar{\tau}_{\beta}(s)-z_{*}\right)\right\rangle \mathbf{C}^{-1} \overline{\mathbf{C}}_{\beta}$

$\times\left(\overline{\mathbf{A}}^{\mathrm{T}} \operatorname{Im}[\mathbf{d}]+\overline{\mathbf{B}}^{\mathrm{T}} \operatorname{Im}[\mathbf{c}]\right) h_{n}(s) d s$. 
Based on Eqs (3), (9) and (31), one can derive the following Somigliana type integral identity for extended displacement at the arbitrary point $\xi$ of thermoelastic half-space:

$\mathbf{u}(\xi)=2 \operatorname{Re}\left\{\operatorname{Af}\left(Z_{*}(\xi)\right)+\mathbf{c} g\left(Z_{t}(\xi)\right)\right\}=\int_{\Gamma}\left[\mathbf{U}^{h s}(\mathbf{x}, \xi) \mathbf{t}(\mathbf{x})\right.$

$\left.-\mathbf{T}^{\mathrm{hs}}(\mathbf{x}, \xi) \mathbf{u}(\mathbf{x})+\mathbf{r}^{h s}(\mathbf{x}, \xi) \theta(\mathbf{x})+\mathbf{v}^{h s}(\mathbf{x}, \xi) h_{n}(\mathbf{x})\right] d s(\mathbf{x})$.

Here, the kernels are defined as:

$\mathbf{U}^{h s}(\mathbf{x}, \boldsymbol{\xi})=\frac{1}{\pi} \operatorname{Im}\left[\mathbf{A}\left\langle\ln Z_{*}(\mathbf{x}-\xi)\right\rangle \mathbf{A}^{\mathrm{T}}\right.$

$\left.+\mathbf{A} \sum_{\beta=1}^{3}\left\langle\ln \left(\bar{Z}_{\beta}(\mathbf{x})-Z_{*}(\xi)\right)\right) \mathbf{C}^{-1} \overline{\mathbf{C}}_{\beta} \overline{\mathbf{A}}^{\mathrm{T}}\right]$

$\mathbf{v}^{h s}(\mathbf{x}, \boldsymbol{\xi})=-\frac{1}{\pi k_{t}} \operatorname{Im}\left[\mathbf{A}\left\langle f^{*}\left(Z_{*}(\mathbf{x}-\xi)\right)\right\rangle\left(\mathbf{A}^{\mathrm{T}} \operatorname{Im}[\mathbf{d}]\right.\right.$

$\left.\left.-\mathbf{B}^{\mathrm{T}} \operatorname{Im}[\mathbf{c}]\right)\right]-\frac{1}{\pi k_{t}} \operatorname{Im}\left[\sum_{\beta=1}^{3} \mathbf{A}\left\langle f^{*}\left(\bar{Z}_{\beta}(\mathbf{x})-Z_{*}(\xi)\right)\right\rangle \mathbf{C}^{-1} \overline{\mathbf{C}} \mathbf{I}_{\beta}\right.$

$\left.\times\left(\overline{\mathbf{A}}^{\mathrm{T}} \operatorname{Im}[\mathbf{d}]+\overline{\mathbf{B}}^{\mathrm{T}} \operatorname{Im}[\mathbf{c}]\right)\right]-\frac{1}{2 \pi k_{t}} \operatorname{Re}\left[\mathbf{c}\left[f^{*}\left(Z_{t}(\mathbf{x}-\xi)\right)\right.\right.$

$\left.\left.+f^{*}\left(\bar{Z}_{t}(\mathbf{x})-Z_{t}(\xi)\right)\right]\right]+\frac{1}{\pi k_{t}} \operatorname{Re}\left[\mathbf{A}\left\langle f^{*}\left(\bar{Z}_{t}(\mathbf{x})-Z_{*}(\xi)\right)\right\rangle\right.$

$\left.\times\left(\mathbf{m}+\mathbf{C}^{-1} \overline{\mathbf{C}} \overline{\mathbf{m}}\right)\right]$;

$\mathbf{T}^{h s}(\mathbf{x}, \xi)=\frac{1}{\pi} \operatorname{Im}\left[\mathbf{A}\left(\frac{n_{2}(\mathbf{x})-p_{*} n_{1}(\mathbf{x})}{Z_{*}(\mathbf{x}-\xi)}\right) \mathbf{B}^{\mathrm{T}}\right.$

$\left.+\mathbf{A} \sum_{\beta=1}^{3}\left\langle\frac{n_{2}(\mathbf{x})-\bar{p}_{\beta} n_{1}(\mathbf{x})}{\bar{z}_{\beta}(\mathbf{x})-Z_{*}(\xi)}\right\rangle \mathbf{I}_{\beta} \mathbf{C}^{-1} \overline{\mathbf{C}}^{\mathbf{B}}\right]$ and

$\mathbf{r}_{i}^{h s}(\mathbf{x}, \xi)=-\frac{1}{\pi} \operatorname{Im}\left[\mathbf{A}\left\langle\ln Z_{*}(\mathbf{x}-\xi)\right\rangle\left(\mathbf{A}^{\mathrm{T}} \operatorname{Re}\left\{\mathbf{d}\left(n_{2}-p_{t} n_{1}\right)\right\}\right.\right.$

$\left.\left.+\mathbf{B}^{\mathrm{T}} \operatorname{Re}\left\{\mathbf{c}\left(n_{2}-p_{t} n_{1}\right)\right\}\right)\right]-\frac{1}{\pi} \operatorname{Im} \sum_{\beta=1}^{3}\left[\mathbf{A}\left\langle\ln \left(\bar{Z}_{\beta}(\mathbf{x})-Z_{*}(\xi)\right)\right\rangle\right.$

$\left.\times \mathbf{C}^{-1} \overline{\mathbf{C}}_{\beta}\left(\overline{\mathbf{A}}^{\mathrm{T}} \operatorname{Re}\left\{\mathbf{d}\left(n_{2}-p_{t} n_{1}\right)\right\}+\overline{\mathbf{B}}^{\mathrm{T}} \operatorname{Re}\left\{\mathbf{c}\left(n_{2}-p_{t} n_{1}\right)\right\}\right)\right]$

$-\frac{1}{\pi} \operatorname{Im}\left[\mathbf{A}\left\langle\ln \left(\bar{Z}_{t}(\mathbf{x})-Z_{*}(\xi)\right)\right\rangle \mathbf{A}^{-1} \operatorname{Re}[\mathbf{c}]\left(n_{2}-\bar{p}_{t} n_{1}\right)\right]$

$+\frac{1}{\pi}\left[\mathbf{c}\left[n_{2}-p_{t} n_{1}\right] \ln Z_{t}(\mathbf{x}-\xi)\right.$

$\left.\left.-\left(n_{2}-\bar{p}_{t} n_{1}\right) \ln \left(\bar{Z}_{t}(\mathbf{x})-Z_{*}(\xi)\right)\right]\right]$.

Here, $Z_{*}(\mathbf{x})=x_{1}+p_{*} x_{2}$.

Eqs (5), (13) and (31) yield the following integral formula for the components of the extended stress tensor:

$\boldsymbol{\sigma}_{\mathrm{j}}(\xi)=\left[\sigma_{\mathrm{ij}}(\xi)\right]=2 \operatorname{Re}\left\{\mathbf{B}\left(\delta_{2 j}-\delta_{1 j} P\right) \mathbf{f}^{\prime}\left(Z_{*}(\xi)\right)\right.$

$\left.+\mathbf{d}\left(\delta_{2 j}-\delta_{1 j} p_{t}\right) g^{\prime}\left(Z_{t}(\xi)\right)\right\}=\int_{\Gamma} \mathbf{D}_{j}^{h s}(\mathbf{x}, \xi) \mathbf{t}(\mathbf{x}) d s(\mathbf{x})$

$-\int_{\Gamma} \mathbf{s}_{j}^{h s}(\mathbf{x}, \xi) \mathbf{u}(\mathbf{x}) d s(\mathbf{x})+\int_{\Gamma} \mathbf{q}_{j}^{h s}(\mathbf{x}, \xi) \theta(\mathbf{x}) d s(\mathbf{x})$

$+\int_{\Gamma} \mathbf{w}_{j}^{h s}(\mathbf{x}, \xi) h_{n}(\mathbf{x}) d s(\mathbf{x})$.

Here

$\mathbf{q}_{i j}^{h S}(\mathbf{x}, \xi)=-\frac{1}{\pi} \operatorname{Im}\left\{\mathbf{B}\left\langle\frac{\delta_{2 j}-p_{*} \delta_{1 j}}{Z_{*}(\mathbf{x}-\xi)}\right\rangle\right.$

$\left.\times\left(\mathbf{A}^{\mathrm{T}} \operatorname{Re}\left\{\mathbf{d}\left(n_{2}-p_{t} n_{1}\right)\right\}+\mathbf{B}^{\mathrm{T}} \operatorname{Re}\left\{\mathbf{c}\left(n_{2}-p_{t} n_{1}\right)\right\}\right)\right\}$

$\frac{1}{\pi} \operatorname{Im}\left\{\mathbf{B} \sum_{\beta=1}^{3} \frac{\delta_{2 j}-p_{*} \delta_{1 j}}{\bar{Z}_{\beta}(\mathbf{x})-Z_{*}(\boldsymbol{\xi})} \mathbf{C}^{-1} \overline{\mathbf{C}}_{\beta}\right.$

$\left.\times\left(\overline{\mathbf{A}}^{\mathrm{T}} \operatorname{Re}\left\{\mathbf{d}\left(n_{2}-p_{t} n_{1}\right)\right\}+\overline{\mathbf{B}}^{\mathrm{T}} \operatorname{Re}\left\{\mathbf{c}\left(n_{2}-p_{t} n_{1}\right)\right\}\right)\right\}$

$-\frac{1}{\pi} \operatorname{Im}\left\{\mathbf{B}\left(\frac{\delta_{2 j}-p_{*} \delta_{1 j}}{\bar{Z}_{\beta}(\mathbf{x})-Z_{*}(\xi)}\right) \mathbf{A}^{-1} \operatorname{Re}[\mathbf{c}]\left(n_{2}-\bar{p}_{t} n_{1}\right)\right\}$ $-\frac{1}{2 \pi} \operatorname{Im}\left\{\mathbf{d}\left(\delta_{2 j}-p_{*} \delta_{1 j}\right)\left[\frac{\delta_{2 j}-p_{*} \delta_{1 j}}{Z_{*}(\mathbf{x}-\xi)}-\frac{\delta_{2 j}-\bar{p}_{*} \delta_{1 j}}{\bar{Z}_{t}(\mathbf{x})-Z_{t}(\xi)}\right]\right\} ;$

$\mathbf{w}_{i j}^{h s}(\mathbf{x}, \boldsymbol{\xi})=\frac{1}{\pi k_{t}} \operatorname{Im}\left[-\mathbf{B}\left\langle\left(\delta_{2 j}-\delta_{1 j} \mathrm{P}_{*}\right) \ln Z_{*}(\mathbf{x}-\xi)\right\rangle\right.$

$\left.\times\left(\mathbf{B}^{\mathrm{T}} \operatorname{Im}[\mathbf{c}]+\mathbf{A}^{\mathrm{T}} \operatorname{Im}[\mathbf{d}]\right)\right]$

$+\frac{1}{\pi k_{t}} \operatorname{Im}\left[\sum_{\beta=1}^{3} \mathbf{B}\left\langle\left(\delta_{2 j}-\delta_{1 j} \mathrm{P}_{*}\right) \ln \bar{Z}_{\beta}(\mathbf{x})-Z_{*}(\xi)\right\rangle\right.$

$\left.\times \mathbf{C}^{-1} \overline{\mathbf{C}}_{\beta}\left(\overline{\mathbf{B}}^{\mathrm{T}} \operatorname{Im}[\mathbf{c}]+\overline{\mathbf{A}}^{\mathrm{T}} \operatorname{Im}[\mathbf{d}]\right)\right]$

$\left.-\frac{1}{\pi k_{t}} \operatorname{Re}\left[\sum_{\beta=1}^{3} \mathbf{B}\left\langle\left(\delta_{2 j}-\delta_{1 j} \mathrm{P}_{*}\right) \ln \bar{Z}_{t}(\mathbf{x})-Z_{*}(\xi)\right)\right\rangle \mathbf{A}^{-1} \operatorname{Re}[\mathbf{c}]\right]$

$+\frac{1}{2 \pi k_{t}} \operatorname{Re}\left[\mathbf{d}\left(\delta_{2 j}-\delta_{1 j} \mathrm{P}_{t}\left(\ln Z_{t}(\mathbf{x}-\xi)+\ln \left(\bar{Z}_{t}(\mathbf{x})-\ln Z_{t}(\xi)\right)\right)\right] ;\right.$

$\mathbf{D}_{i j k}^{h s}(\mathbf{x}, \xi)=-\frac{1}{\pi} \operatorname{Im}\left\{\mathbf{B}\left[\mid \frac{\delta_{2 j}-p_{*} \delta_{2 j}}{Z_{*}(\mathbf{x}-\xi)}\right) \mathbf{A}^{\mathrm{T}}\right.$

$\left.\left.+\sum_{\beta=1}^{4}\left(\frac{\delta_{2 j}-p_{*} \delta_{1 j}}{\bar{Z}_{\beta}(\mathbf{x})-Z_{*}(\xi)}\right) \mathbf{C}^{-1} \overline{\mathbf{C}}_{\beta} \overline{\mathbf{A}}^{\mathrm{T}}\right]\right\}$ and

$\mathbf{S}_{i j k}^{h s}(\mathbf{x}, \xi)=\frac{1}{\pi} \operatorname{Im}\left\{\mathbf{B}\left\langle\frac{\left(\delta_{2 j}-p_{*} \delta_{1 j}\right)\left(n_{2}-p_{*} n_{1}\right)}{\left(Z_{*}(\mathbf{x}-\xi)\right)^{2}}\right) \mathbf{B}^{\mathrm{T}}\right\}$

$+\frac{1}{\pi} \operatorname{Im}\left\{\mathbf{B}\left(\delta_{2 j}-p_{*} \delta_{1 j}\right)\right.$

$\left.\times \sum_{\beta=1}^{4}\left\langle\frac{\left(n_{2}-\bar{p}_{*} n_{1}\right)}{\left(\bar{Z}_{\beta}(\mathbf{x})-Z_{*}(\xi)\right)^{2}}\right) \mathbf{C}^{-1} \overline{\mathbf{C}}_{\beta} \overline{\mathbf{B}}^{\mathrm{T}}\right\}$.

Thus, for the problem with boundary conditions in Eqs (8a) and (8c), appropriate integral representations can be obtained.

\section{NUMERICAL EXAMPLES}

Sample problem is considered for an anisotropic (fibreglass) thermoelastic half-plane $x_{2}>0$ under the action of a uniform heat flow directed along its boundary, which contains internal thin, rectilinear thermoelastic isotropic inclusion (Fig. 1). Its length is $l=2 a$ and thickness is $h=0,01 a$, and it is located at a distance $d=a$ to the edge of the half-space. Properties of the half-space are as follows: $E_{1}=55 \mathrm{GPa}, E_{2}=21 \mathrm{GPa}, G_{12}=9.7 \mathrm{GPa}$, $v_{12}=0.25, \quad \alpha_{11}=6.3 \times 10^{-6} \mathrm{~K}^{-1}, \quad \alpha_{22}=2.0 \times 10^{-5} \mathrm{~K}^{-1}$, $k_{11}=3.46 \mathrm{~W} /(\mathrm{m} \cdot \mathrm{K})$ and $k_{22}=0.35 \mathrm{~W} /(\mathrm{m} \cdot \mathrm{K})$. The values of these constants are given in the direction of the main axes of the anisotropy of the material. Inclusion is assumed to be thermally insulated and possesses no thermal expansion.

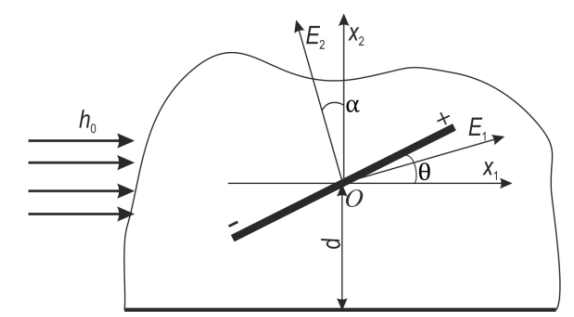

Fig. 1. Thermoelastic anisotropic half-plane with thin inclusion

Figs 2-9 show the relationship between the generalised stress intensity factors (SIF) and the relative rigidity $k=G^{i} / G_{12}\left(G^{i}\right.$ is the shear modulus of inclusion) of the inclusion, under the boundary conditions in Eqs (8a) and (8c) at different values of inclusion inclination angle $\theta$. The normalising SIF $K_{0}=$ 


\section{sciendo}

Heorhiy Sulym, laroslav Pasternak, Mariia Smal, Andrii Vasylyshyn

Mixed Boundary Value Problem for an Anisotropic Thermoelastic Half-Space Containing Thin Inhomogeneities

$a \sqrt{\pi a} \cdot E_{1} \times \alpha_{11} / k_{11} \cdot h_{0}$. Dashed lines show cases when the influence of inclusion bending is excluded from its model. As can be seen from the plot, this effect is significant, and therefore requires careful analysis.

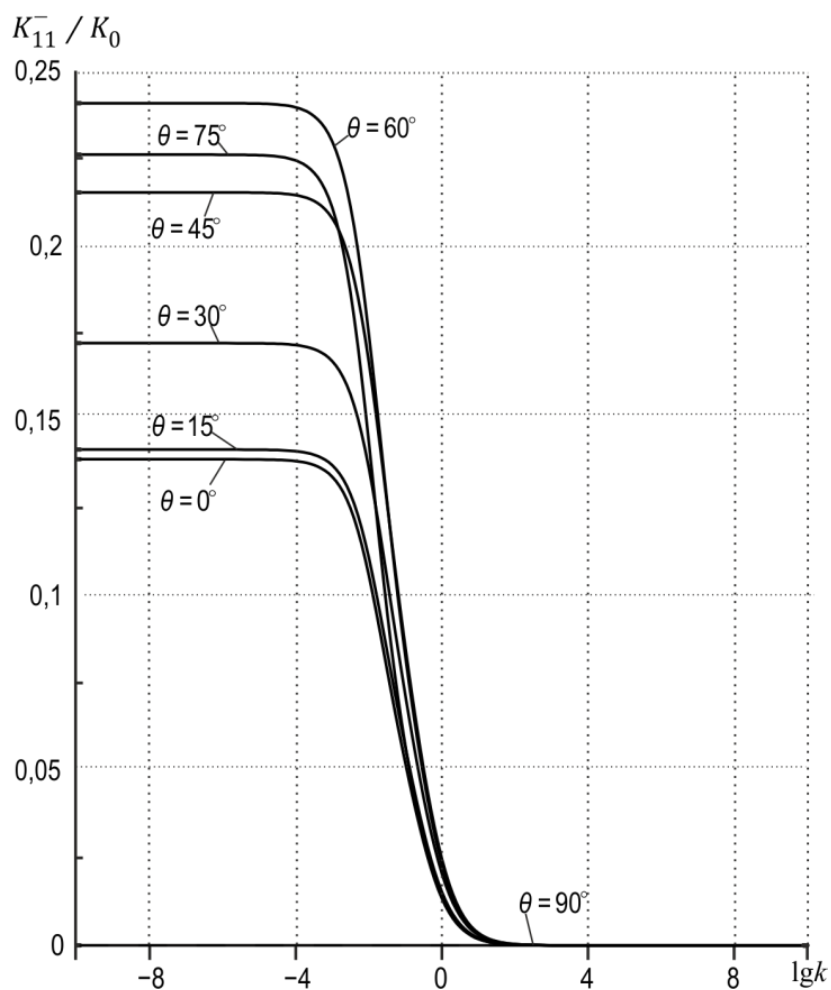

Fig. 2. The relationship between SIF $K_{11}^{-} / K_{0}$ and the generalised stiffness at different values of $\theta$

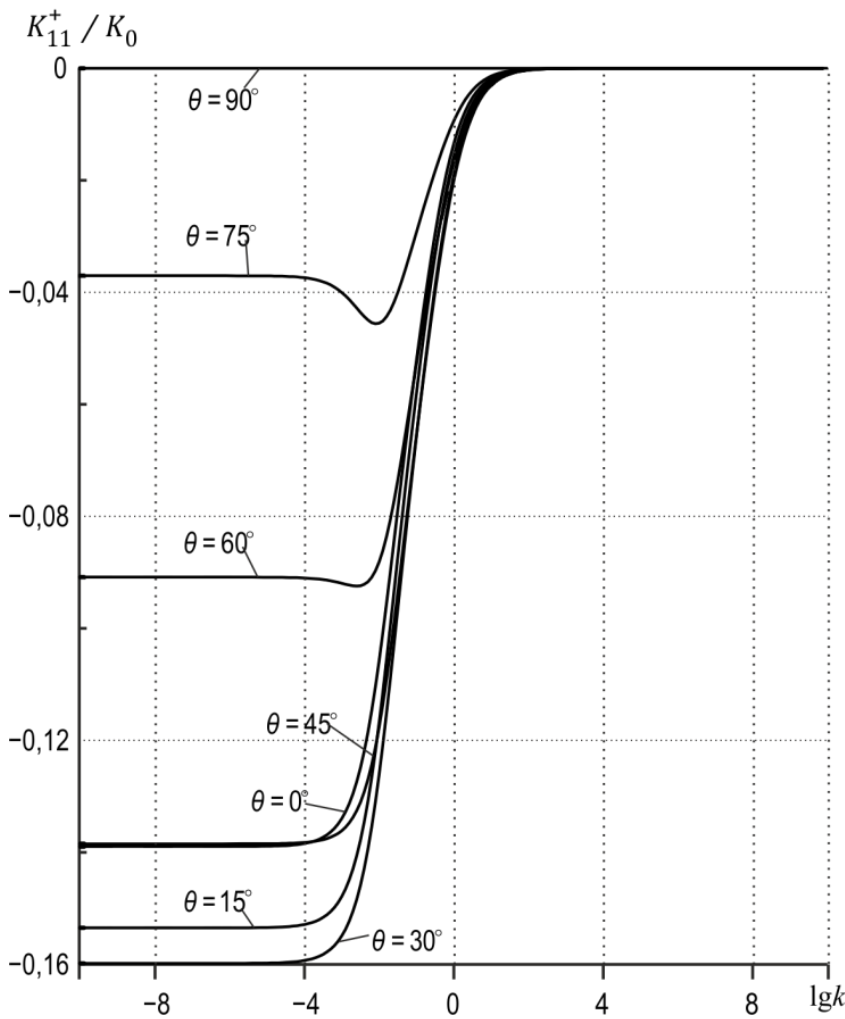

Fig. 3. The relationship between SIF $K_{11}^{+} / K_{0}$ and the generalised stiffness at different values of $\theta$

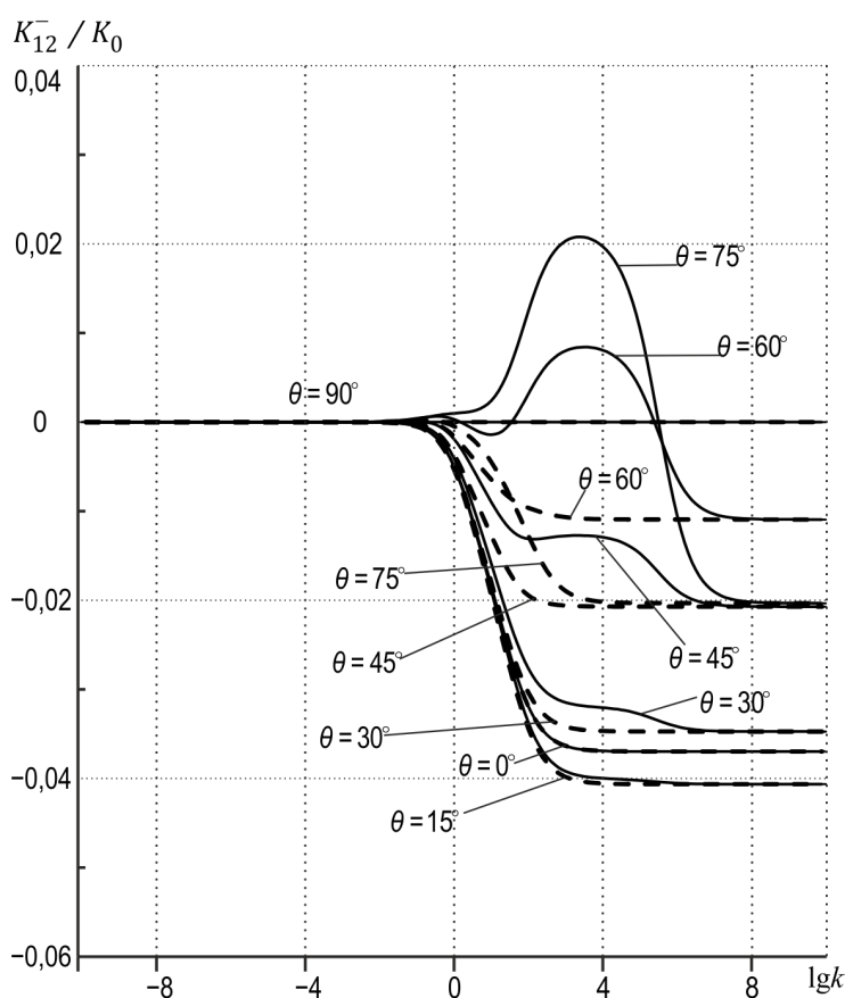

Fig. 4. The relationship between SIF $K_{12}^{-} / K_{0}$ and the generalised stiffness at different values of $\theta$

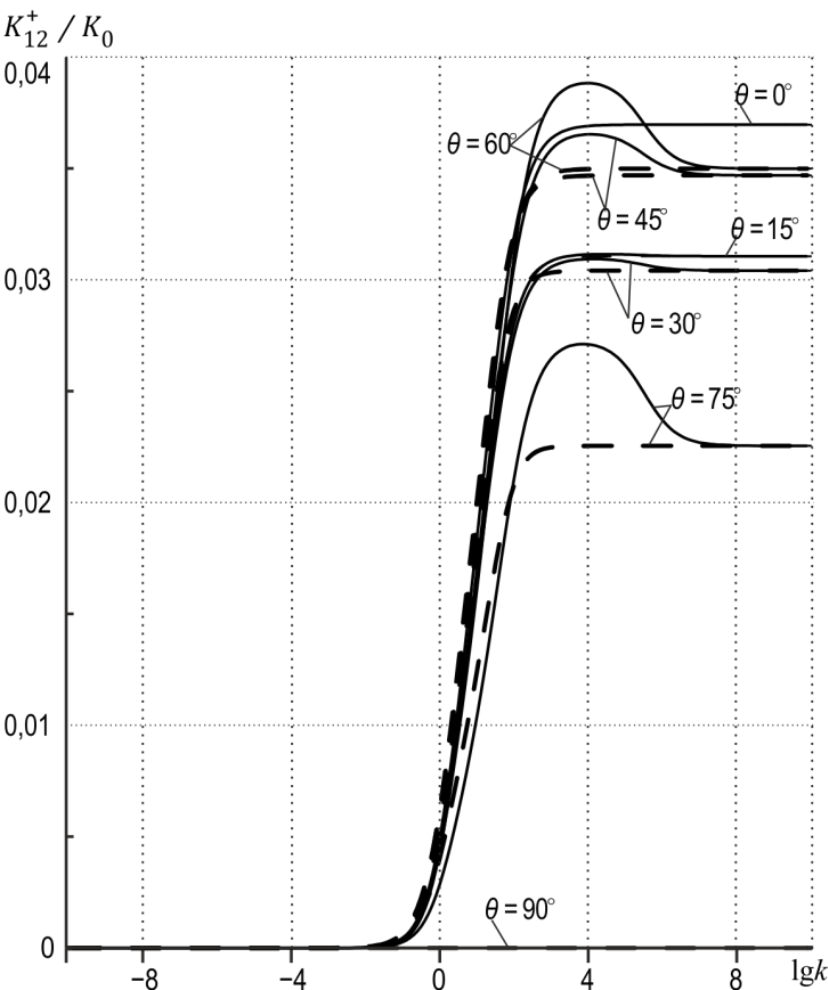

Fig. 5. The relationship between SIF $K_{12}^{+} / K_{0}$ and the generalised stiffness at different values of $\theta$ 


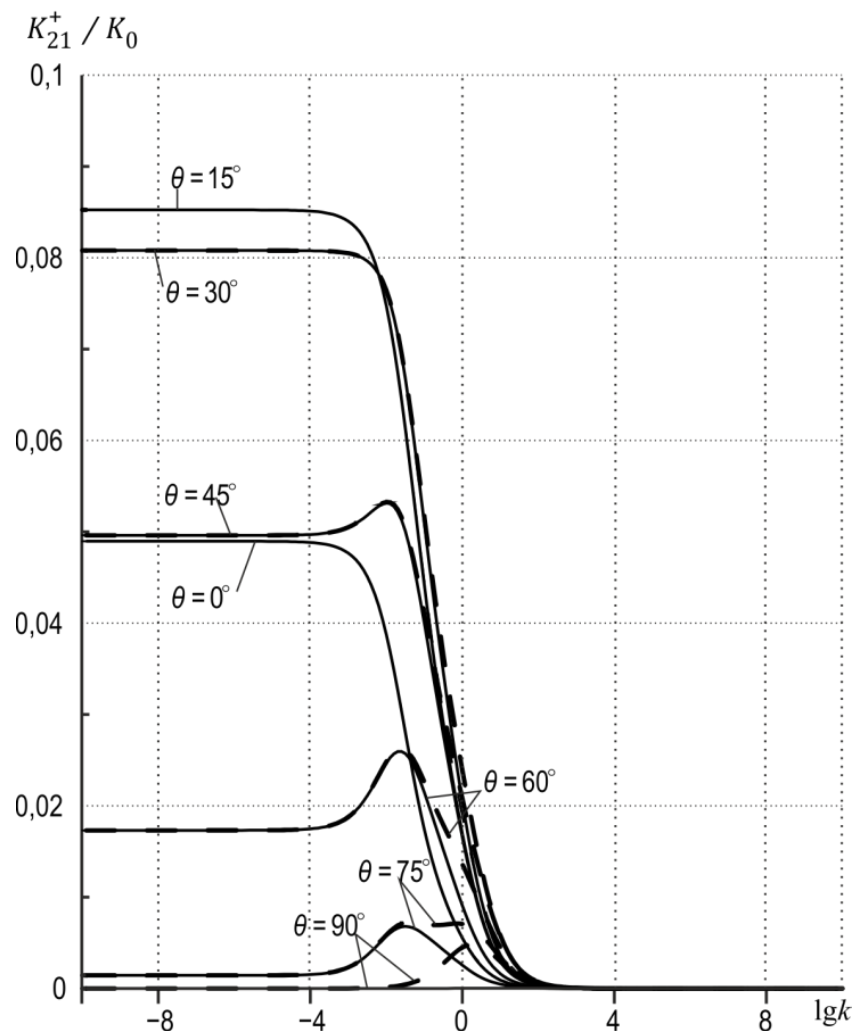

Fig. 6. The relationship between SIF $K_{21}^{+} / K_{0}$ and the generalised stiff ness at different values of $\theta$

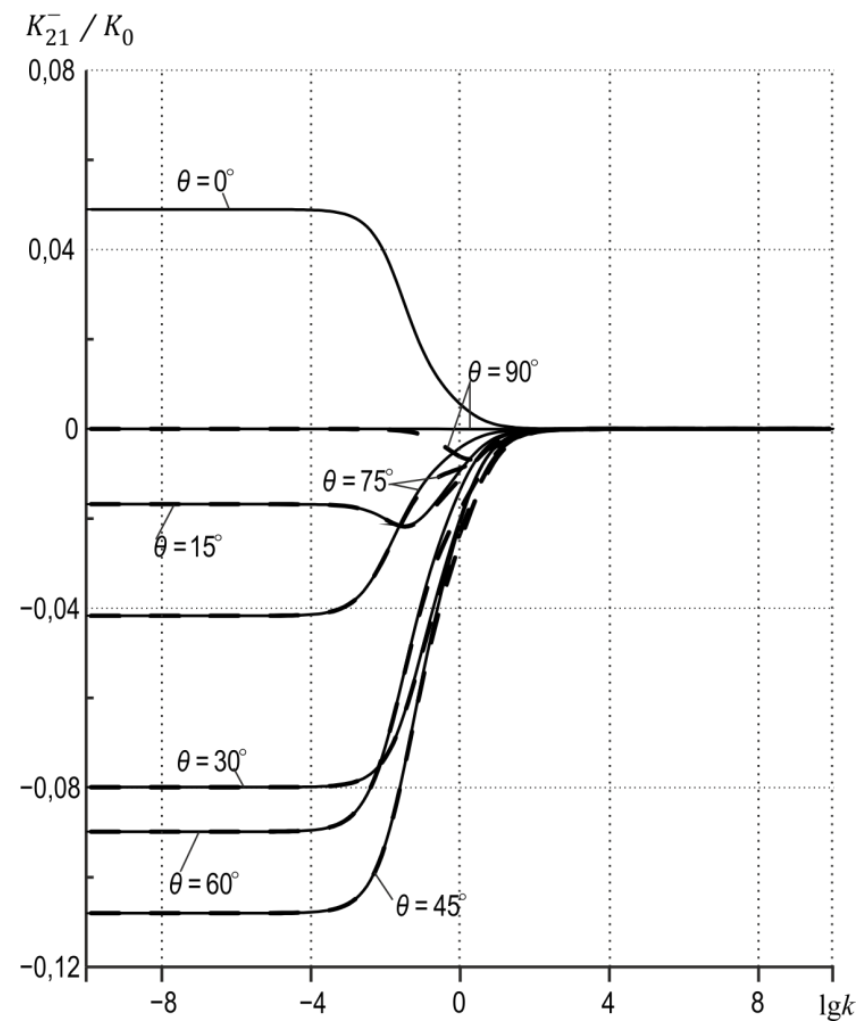

Fig. 7. The relationship between SIF $K_{21}^{-} / K_{0}$ and the generalised stiffness at different values of $\theta$

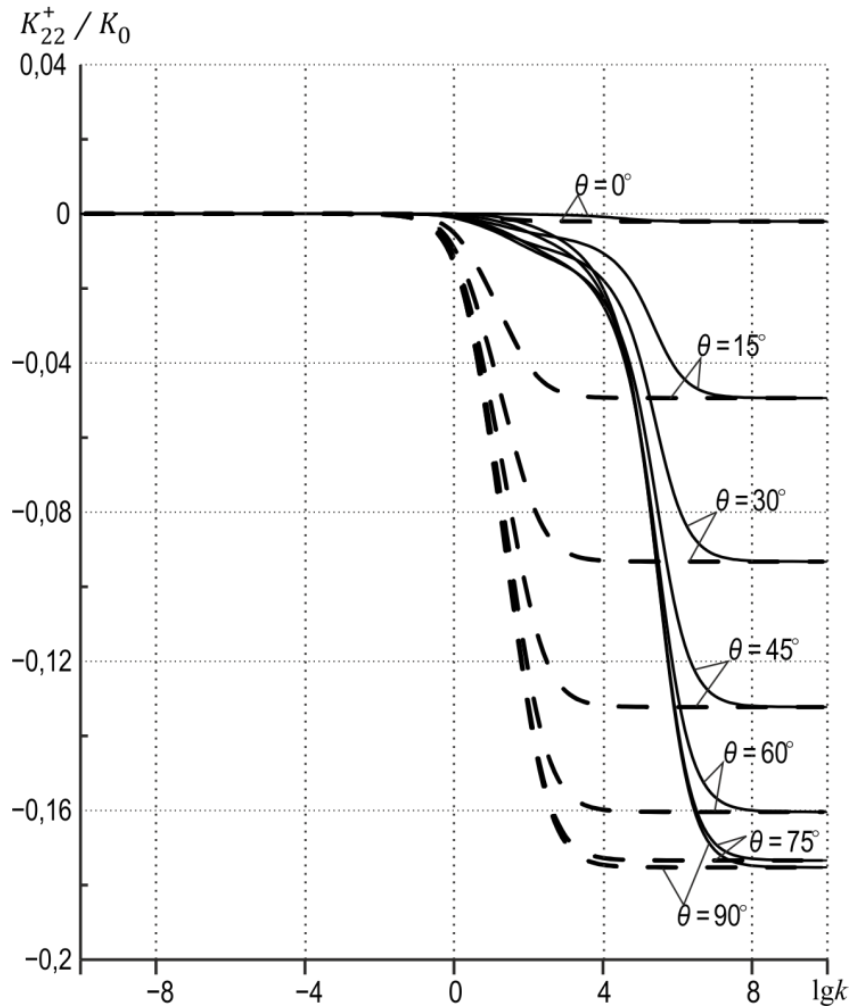

Fig. 8. The relationship between SIF $\frac{K_{22}^{+}}{K_{0}}$ and the generalised stiffness at different values of $\theta$

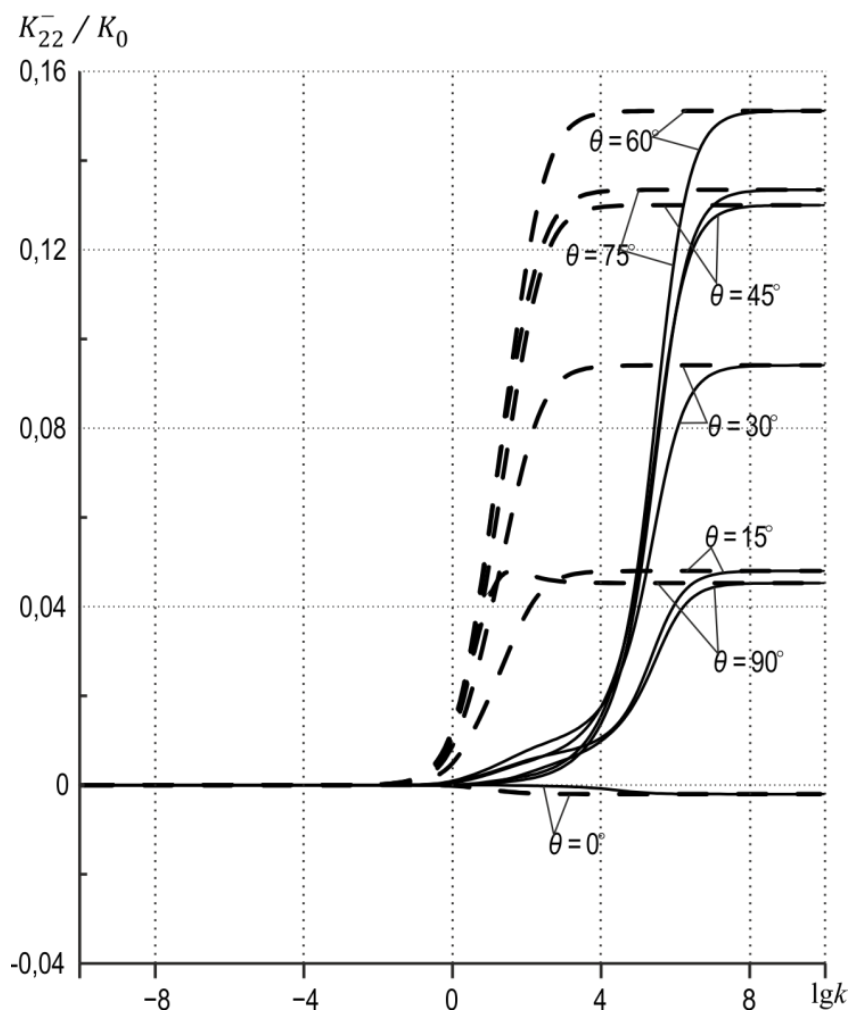

Fig. 9. The relationship between SIF $K_{22}^{-} / K_{0}$ and the generalised stiffness at different values of $\theta$ 
In Figs 2 and 3 , we see that the inclusion bending does not affect the values of the SIFs $K_{11}^{+} / K_{0}$ and $K_{11}^{-} / K_{0}$. It should also be noted that the maximum value of the SIFs $K_{11}^{-} / K_{0}$ is reached when the inclusion is at the angle $\theta=60^{\circ}$ to the boundary of the half-space. At $\lg k<-4$, the values of the SIFs do not change, and at $\lg k>-4$ (except in case of $\theta=75^{\circ}$ ), they monotonically tend to become zero.

In Figs 4-9, it can be seen that the bending of the inclusion significantly affects the values of the SIFs. This is especially noticeable in Fig. 4, when $\theta=75^{\circ}$ and $\theta=60^{\circ}$. As mentioned earlier, this effect requires careful analysis.

One can see in Figs 8 and 9 that below $\lg k=4$, the values of the SIFs grow slowly and above $\lg k>4$, their growth is intense. When the influence of inclusion bending is excluded from consideration, we can see that the SIFs $K_{22}^{+} / K_{0}$ and $K_{22}^{-} / K_{0}$ increase monotonously even at $\lg k=0$ and tend their maximum values faster.

\section{CONCLUSIONS}

The paper presents a simple and straightforward approach for obtaining the Somigliana type integral formulae and corresponding dual boundary integral equations for an anisotropic thermoelastic half-space with mixed boundary conditions on its surface.

Integral equations in conjunction with the scheme of the modified BEM allow solving a number of new problems for the thermoelastic half-plane containing cracks or thin deformable inclusions.

A numerical analysis of the influence of boundary conditions on the half-space boundary and the relative rigidity of the thin inhomogeneity on stress intensity at the inclusions is provided.

\section{REFERENCES}

1. Bozhydarnyk V., Pasternak I., Sulym H., Oliyarnyk N. (2011), BEM approach for the antiplane shear of anisotropic solids containing thin inhomogeneities, Acta mechanica et automatica, 5(4), 11-16.

2. Chen H., Wang Q., Liu G. R., Wang Y., Sun J. (2016), Simulation of thermoelastic crack problems using singular edge-based smoothed finite element method, International Journal of Mechanical Sciences, 115-116, 123-134.

3. Hou P.F. (2011), 2D general solution and fundamental solution for orthotropic thermoelastic materials, Engineering Analysis with Boundary Elements, 35, 56-60.

4. Hwu C. (2010), Anisotropic elastic plates, Springer, London.

5. Li X.Y. (2012), Exact fundamental thermo-elastic solutions of a transversely isotropic elastic medium with a half infinite plane crack International Journal of Mechanical Sciences, 59(1), 83-94.

6. Mukherjee Y.X. (1999), Thermoelastic fracture mechanics with regularized hypersingular boundary integral equations, Engineering Analysis with Boundary Elements, 23, 89-96.

7. Pasternak I., Pasternak R., Sulym H. (2013), Boundary integral equations for 2D thermoelasticity of a half-space with cracks and thin inclusions, Engineering Analysis with Boundary Elements, 37, 15141523.

8. Pasternak I. (2012), Boundary integral equations and the boundary element method for fracture mechanics analysis in 2D anisotropic thermoelasticity, Engineering Analysis with Boundary Elements, 36(12), 1931-1941.

9. Qin Q. (1999), Thermoelectroelastic analysis of cracks in piezoelectric half-plane by BEM, Computational Mechanics, 23, 353-360.
10. Şeremet V. (2011), Deriving exact Green's functions and integral formulas for a thermoelastic wedge, Engineering Analysis with Boundary Elements, 35(3), 527-532.

11. Sherief H.H., Abd El-Latief A.M. (2014), Application of fractional order theory of thermoelasticity to a $2 \mathrm{D}$ problem for a half-space, Applied Mathematics and Computation, 248, 584-592.

12. Shiah Y.C. (2000), Fracture mechanics analysis in 2-D anisotropic thermoelasticity using BEM, CMES, 1(3), 91-99.

13. Sulym H.T. (2007), Bases of mathematical theory of thermo-elastic equilibrium of solids containing thin inclusions, Research and Publishing center of NTSh, 2007 (in Ukrainian).

14. Tokovyy Y., Ma C-C. (2009), An explicit-form solution to the plane elasticity and thermoelasticity problems for anisotropic and inhomogeneous solids, Int J Solids Struct, 46(21), 3850-9.

15. Woo H-G., Li H. (2011), Advanced functional materials, Springer, London.

16. Wu W-L. (2009), Dual Boundary Element Method Applied to Antiplane Crack Problems, Mathematical Problems in Engineering, doi:10.1155/2009/132980.

17. Yang W., Zhou Q., Zhai Yu, Lyu D., Huang Y., Wang J., Jin X., Keer M.L., Wang Q.J. (2019), Semi-analytical solution for steady state heat conduction in a heterogeneous half space with embedded cuboidal inhomogeneity, International Journal of Thermal Sciences, 139, 326-338.

The present paper is financially supported by the Ministry of Science and Higher Education of Poland (research project No. S/WM/4/2017) and realised in Bialystok University of Technology. 